\title{
Reduction of titanium oxide in the presence of nickel by nonequilibrium hydrogen gas
}

\section{$\operatorname{AUTHOR}(\mathrm{S}):$}

Sekimoto, Hidehiro; Uda, Tetsuya; Nose, Yoshitaro; Sato, Shigeo; Kakiuchi, Hiroaki; Awakura, Yasuhiro

\section{CITATION:}

Sekimoto, Hidehiro ... [et al]. Reduction of titanium oxide in the presence of nickel by nonequilibrium hydrogen gas. JOURNAL OF MATERIALS RESEARCH 2009, 24(7): 2391-2399

\section{ISSUE DATE:}

2009-07

URL:

http://hdl.handle.net/2433/109947

RIGHT:

(C) 2009, Materials Research Societyiety 


\title{
Reduction of titanium oxide in the presence of nickel by nonequilibrium hydrogen gas
}

\author{
Hidehiro Sekimoto, ${ }^{\text {a) }}$ Tetsuya Uda, and Yoshitaro Nose \\ Department of Materials Science and Engineering, Graduate School of Engineering, \\ Kyoto University, Kyoto 606-8501, Japan \\ Shigeo Sato \\ Institute for Materials Research, Tohoku University, Sendai 980-8577, Japan \\ Hiroaki Kakiuchi \\ Department of Precision Science and Technology, Division of Precision Science \& \\ Technology and Applied Physics, Graduate School of Engineering, \\ Osaka University, Osaka 565-0871, Japan \\ Yasuhiro Awakura \\ Department of Materials Science and Engineering, Graduate School of Engineering, \\ Kyoto University, Kyoto 606-8501, Japan
}

(Received 16 December 2008; accepted 22 April 2009)

We investigated the reduction of $\mathrm{TiO}_{2}$ in the presence of $\mathrm{Ni}$ by nonequilibrium hydrogen gas, including low-temperature hydrogen plasma at $800{ }^{\circ} \mathrm{C}$ and supercooled monatomic hydrogen at $1000{ }^{\circ} \mathrm{C}$. $\mathrm{TiO}_{2}$ was reduced to $\mathrm{Ti}_{2} \mathrm{O}_{3}$, which is not in equilibrium phase, by low-temperature hydrogen plasma. The results of $\mathrm{x}$-ray diffraction and energy dispersive $\mathrm{x}$-ray analysis in experiments at $1000{ }^{\circ} \mathrm{C}$ indicate that the thermodynamical reduction potential of supercooled monatomic hydrogen is almost the same as atmospheric hydrogen gas. However, the wide $\mathrm{Ti}_{3} \mathrm{O}_{5}$ layer formed only in the case of the reduction at $1000{ }^{\circ} \mathrm{C}$ by supercooled monatomic hydrogen. With these experimental facts, we speculate that the reduction mechanism by nonequilibrium hydrogen consists of two steps; the releasing energy process and the relaxation process. We can explain the difference of reduction products by nonequilibrium hydrogen gas on the assumption of the rate of the relaxation process between 800 and $1000{ }^{\circ} \mathrm{C}$.

\section{INTRODUCTION}

Titanium alloys are attractive materials because they have various unique properties. $\mathrm{Ni}-\mathrm{Ti}$ alloy shows shape memory and a superelasticity effect. The $\mathrm{Nb}-\mathrm{Ti}$ alloy is a superconducting material. Some titanium alloys show excellent corrosion resistance and heat resistance. ${ }^{1,2}$ However, such materials are expensive. One of the reasons is that they are made from an alloy metal and pure titanium, which is an expensive material because of its high production cost. The major production process of titanium is the Kroll process, which consists of carbochlorination of $\mathrm{TiO}_{2}$, purification of $\mathrm{TiCl}_{4}$, magnesiothermic reduction of $\mathrm{TiCl}_{4}$ to metallic titanium, and vacuum distillation of $\mathrm{MgCl}_{2}$ and excess $\mathrm{Mg}$ from metallic titanium. This process has problems of process complication, low productivity of titanium, and high energy consumption. ${ }^{3,4}$ Therefore, direct reduction of titanium oxide to titanium alloy has an advantage of simplicity. ${ }^{3}$

\footnotetext{
a) Address all correspondence to this author.

e-mail: hidehiro.sekimoto@t14.mbox.media.kyoto-u.ac.jp

DOI: 10.1557/JMR.2009.0286
}

Reduction by hydrogen gas may be the simplest process because the only by-product is water vapor. In this study, the reduction of titanium oxide in the presence of nickel as the alloying element to titanium alloy by hydrogen gas was investigated.

\section{THERMODYNAMICS OF Ti-Ni-O TERNARY SYSTEM}

Figure 1(a) shows the equilibrium chemical potential of oxygen, $\mathrm{R} T \ln p_{\mathrm{O}_{2}}$, in equilibrium with some titanium oxides as a function of temperature, $T$, under the conditions that activities of substances in condensed phases are unity. Here, $\mathrm{R}$ is the gas constant, and $p_{i}$ is the partial pressure of chemical species, $i$. RT $\ln p_{\mathrm{O}_{2}}$ in equilibrium with $\mathrm{H}_{2}$ of $1 \mathrm{~atm}$ and $\mathrm{H}_{2} \mathrm{O}$ of $1 \mathrm{~atm}$ or $10^{-4}$ atm are also shown in Fig. 1. Figure $1(\mathrm{~b})$ shows $\mathrm{R} T \ln p_{\mathrm{O}_{2}}$ in equilibrium with titanium oxides and $\mathrm{Ti}-\mathrm{Ni}$ alloys and $\mathrm{R} T \ln p_{\mathrm{O}_{2}}$ in equilibrium with $\mathrm{H}$ (monatomic hydrogen) of $1 \mathrm{~atm}$ and $\mathrm{H}_{2} \mathrm{O}$ of $1 \mathrm{~atm}$. The lines in Fig. 1(b) will be discussed later. From the figure, when $\mathrm{R} T \ln p_{\mathrm{O}_{2}}$ in equilibrium with $\mathrm{H}_{2}$ and $\mathrm{H}_{2} \mathrm{O}$ is lower than that with $\mathrm{Ti}$ and $\mathrm{TiO}, \mathrm{TiO}_{2}$ can be reduced to $\mathrm{Ti}$ by hydrogen. But the 

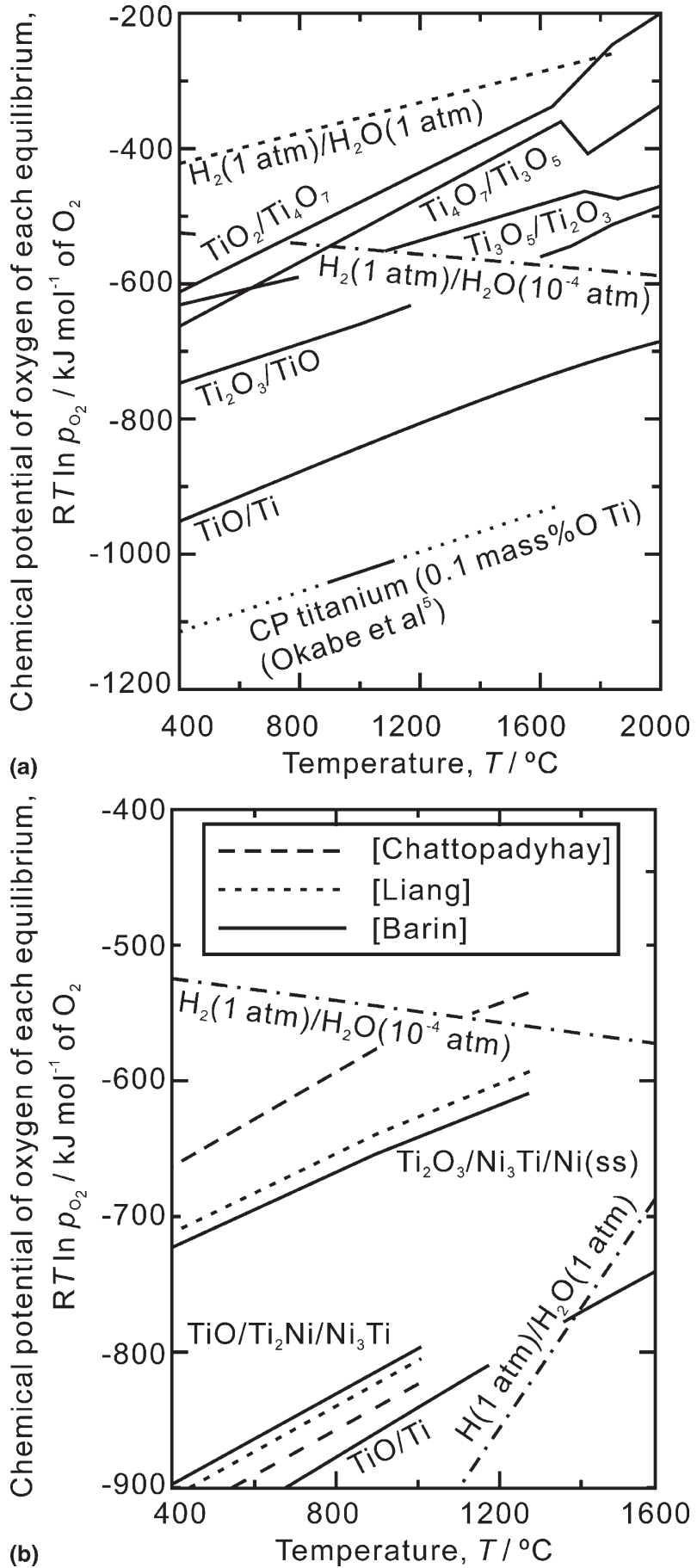

FIG. 1. (a) Equilibrium chemical potential of oxygen, in equilibrium with some titanium oxides, as a function of temperature. Dashed and dashed-dotted lines show chemical potential of oxygen in the $\mathrm{H}_{2} /$ $\mathrm{H}_{2} \mathrm{O}$ equilibrium, where $p_{\mathrm{H}_{2} \mathrm{O}}$ is $1 \mathrm{~atm}$ and $10^{-4}$ atm, respectively. The line for $\mathrm{CP}$ titanium is calculated from the thermodynamic data reported by Okabe et al. ${ }^{5}$ (b) Equilibrium chemical potential of oxygen in equilibrium with titanium oxides and $\mathrm{Ti}-\mathrm{Ni}$ alloys and that in the $\mathrm{H} / \mathrm{H}_{2} \mathrm{O}$ equilibrium, where $p_{\mathrm{H}}$ and $p_{\mathrm{H}_{2} \mathrm{O}}$ are $1 \mathrm{~atm}$.

figure indicates that it is very difficult to obtain Ti by hydrogen reduction. We empirically know that it is not easy to decrease the partial pressure of $\mathrm{H}_{2} \mathrm{O}, P_{\mathrm{H}_{2} \mathrm{O}}$, in the gas boundary layer near titanium oxides below $10^{-4}$ atm during hydrogen reduction. Consequently, we have to give up on reducing titanium oxide to pure metallic titanium. Instead of obtaining pure titanium, we tried to reduce titanium oxide to an alloy.

The reduction of $\mathrm{TiO}_{2}$ to $\mathrm{Ti}$ by hydrogen is expressed by Eq. (1), and its Gibbs energy change, $\Delta G$, is represented by Eq. (2).

$$
\begin{gathered}
\mathrm{TiO}_{2}+2 \mathrm{H}_{2} \rightarrow \mathrm{Ti}+2 \mathrm{H}_{2} \mathrm{O}, \\
\Delta G=\Delta G^{\circ}+\mathrm{R} T \ln \frac{a_{\mathrm{Ti}} p_{\mathrm{H}_{2} \mathrm{O}}^{2}}{a_{\mathrm{TiO}_{2}} p_{\mathrm{H}_{2}}^{2}}=\Delta G^{\circ}+2.303 \mathrm{R} T \\
\times\left(\log a_{\mathrm{Ti}}+2 \log p_{\mathrm{H}_{2} \mathrm{O}}-\log a_{\mathrm{TiO}_{2}}-2 \log p_{\mathrm{H}_{2}}\right),
\end{gathered}
$$

where $\Delta G^{\circ}$ is the standard Gibbs energy change. The value of $\Delta G$ is positive under normal conditions, but might become negative by decreasing $a_{\mathrm{Ti}}$ and $p_{\mathrm{H}_{2} \mathrm{O}}$, or increasing $a_{\mathrm{TiO}_{2}}$ and $p_{\mathrm{H}_{2}}$ It is difficult to decrease $p_{\mathrm{H}_{2} \mathrm{O}}$ to below $10^{-4}$ atm, and to increase $a_{\mathrm{TiO}_{2}}$ to above unity. Thus, to make the value of $\Delta G$ negative, it is necessary to decrease $a_{\mathrm{Ti}}$ and/or increase $p_{\mathrm{H}_{2}}$.

The activity of titanium in alloy is lower than that of pure titanium, which means that alloying with other metal leads to a decrease in $a_{\mathrm{Ti}}$. In the presence of an alloy element, which has a strong affinity with titanium, titanium oxide might be reduced to titanium alloy in reduction by hydrogen gas. In fact, the reduction of $\mathrm{TiO}_{2}$ in the presence of platinum by hydrogen gas has been confirmed by Meschter and Worrel, ${ }^{6}$ and Shioi et al. ${ }^{7}$ Whether or not the reaction proceeds depends on the affinity between titanium and the alloy element. We estimate the affinity from the formation enthalpy of the titanium alloy. Nickel is selected as an appropriate element for this study because the affinity between titanium and nickel is relatively strong; the formation enthalpy of $\mathrm{Ni}_{3} \mathrm{Ti}$ is $-152.2 \mathrm{~kJ} \mathrm{~mol}^{-1}$.

As a way of increasing $p_{\mathrm{H}_{2}}$, the use of nonequilibrium hydrogen gas, including hydrogen plasma or supercooled monatomic hydrogen, is considered. Some researchers attempted reduction of titanium oxide by hydrogen plasma. ${ }^{8-10}$ Although they have not obtained metallic titanium with hydrogen plasma reduction, Huet et al. ${ }^{10}$ reported that $\mathrm{TiO}_{2}$ could be reduced to $\mathrm{TiO}$ at about $800{ }^{\circ} \mathrm{C}$ by low-temperature hydrogen plasma. ${ }^{9}$ $\mathrm{TiO}$ cannot be obtained through reduction of $\mathrm{TiO}_{2}$ by atmospheric hydrogen gas under normal conditions. Therefore, these results clearly indicate that low-temperature hydrogen plasma is a stronger reductant than atmospheric hydrogen gas.

From the background described previously, we were motivated to examine the combination of these ways to obtain titanium alloy from a mixture of titanium oxide and nickel. In this study, we thus investigate the reduction of titanium oxide by low-temperature hydrogen 
plasma or by supercooled monatomic hydrogen in the presence of nickel.

The phase diagram of the Ti-Ni-O ternary system at $927^{\circ} \mathrm{C}$ is shown in Fig. 2(a). ${ }^{11}$ Figure 2(b) is a schematic illustration showing the equilibrium phase relationship between titanium oxides and nickel in detail. Ni(ss) means solid solution of nickel phase. It does not have solubility of titanium when $\mathrm{Ni}$ (ss) and $\mathrm{Ti}_{4} \mathrm{O}_{7}$ are in equilibrium, whereas $\mathrm{Ni}(\mathrm{ss})$ has solubility of 3 at.\% titanium when $\mathrm{Ni}(\mathrm{ss})$ and $\mathrm{Ti}_{3} \mathrm{O}_{5}$ are in equilibrium. The upper limit of the solubility of titanium in $\mathrm{Ni}(\mathrm{ss})$ is 11 at.\%, when $\mathrm{Ni}(\mathrm{ss}), \mathrm{Ti}_{2} \mathrm{O}_{3}$, and $\mathrm{Ni}_{3} \mathrm{Ti}$ are in equilibrium. On the other hand, we can know the extent of activity of titanium in a system from the concentration of $\mathrm{Ti}$ in $\mathrm{Ni}(\mathrm{ss})$. This information will be a

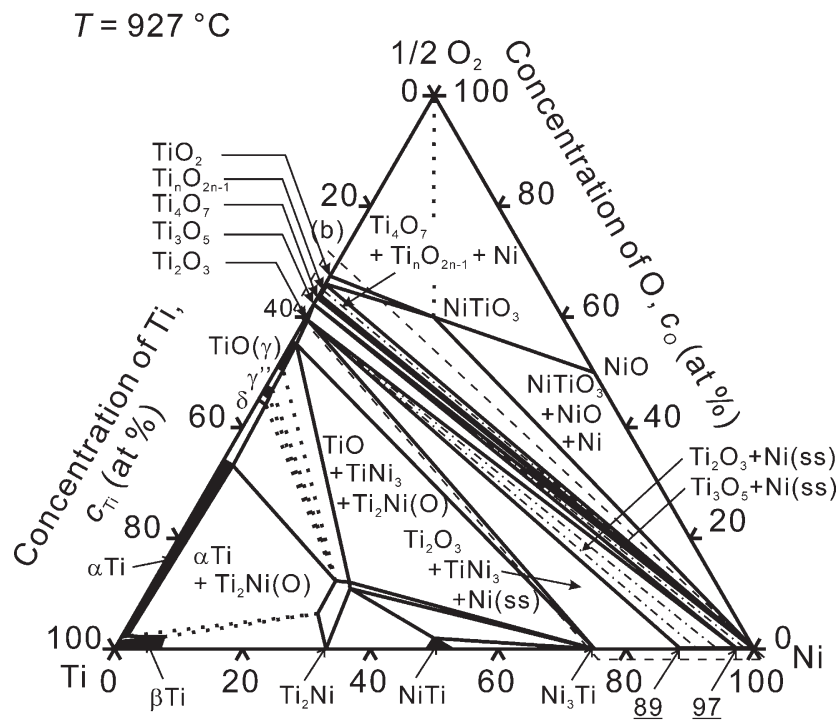

(a)

Concentration of $\mathrm{Ni}, \mathrm{c}_{\mathrm{Ni}}($ at \%)

$T=927^{\circ} \mathrm{C}$ Concentration of $\mathrm{Ti}, \mathrm{C}_{\mathrm{Ti}}($ at \%)

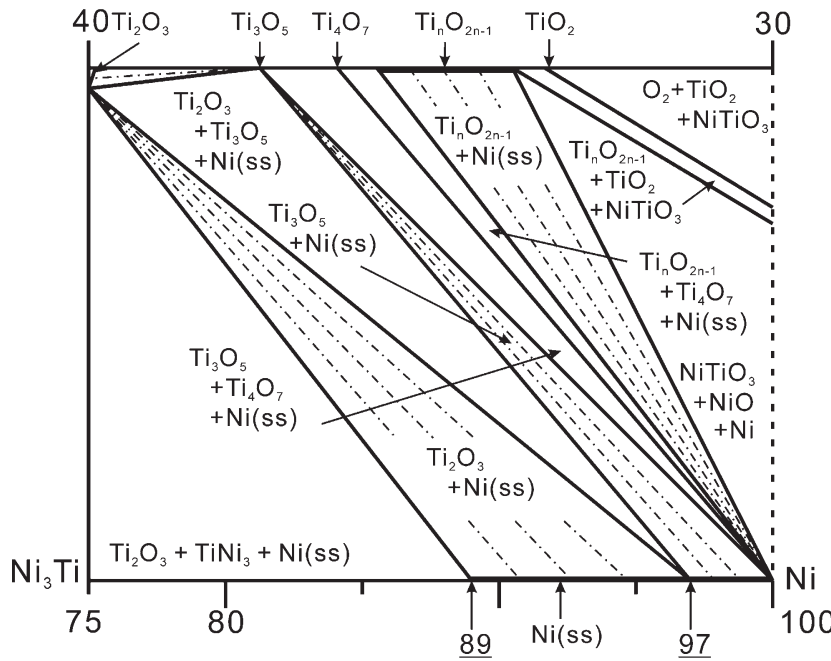

(b)

Concentration of $\mathrm{Ni}, \mathrm{C}_{\mathrm{Ni}}($ at $\%)$

FIG. 2. (a) Isothermal cross section of phase diagram of Ti-Ni-O ternary system at $927{ }^{\circ} \mathrm{C} .{ }^{10}$ (b) Schematic view of phase relation between titanium oxides and $\mathrm{Ni}(\mathrm{ss})$, as shown by dashed line in (a). key for analyzing the reduction potential of supercooled monatomic hydrogen in Sec. V. A.

The chemical potential diagram of the Ti-Ni-O ternary system is shown in Fig. 3. This diagram was made using the standard Gibbs energy of formation at $1000{ }^{\circ} \mathrm{C} .{ }^{11}$ Three axes show the chemical potentials of titanium, nickel, and oxygen. Here, each chemical potential can be represented by $\log a_{\mathrm{Ti}}, \log a_{\mathrm{Ni}}$, and $\log p_{\mathrm{O}_{2}}$ in Fig. 3 . It is assumed that each solid phase has no solubility of other elements except for $\alpha$-Ti, $\beta$-Ti, and $\mathrm{Ni}(\mathrm{ss})$. The $\alpha$-Ti

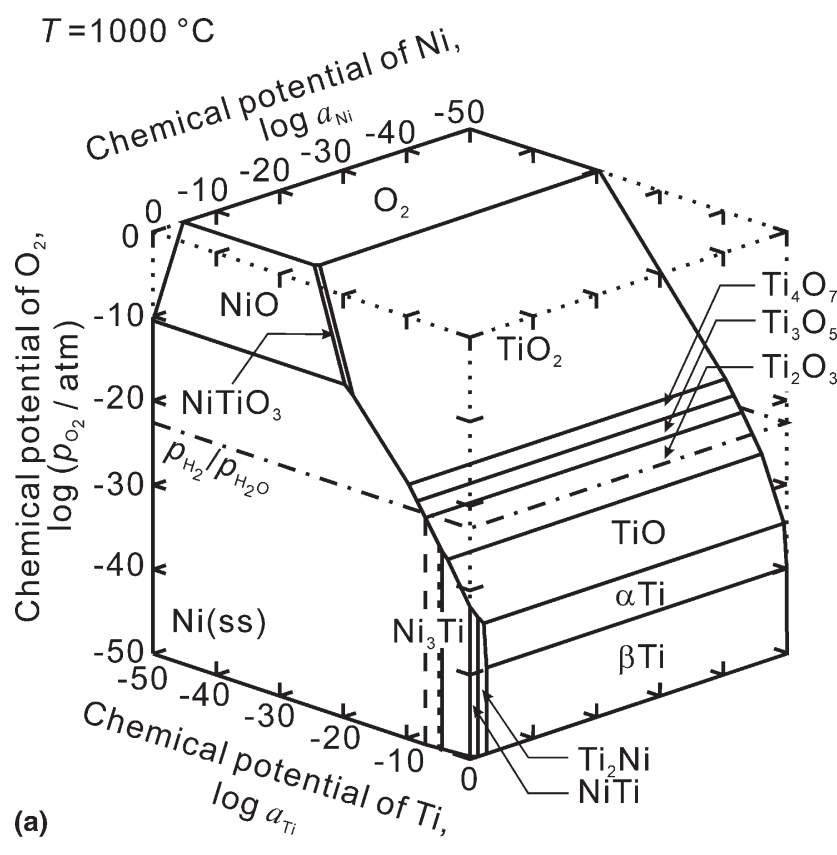

$T=1000^{\circ} \mathrm{C}$

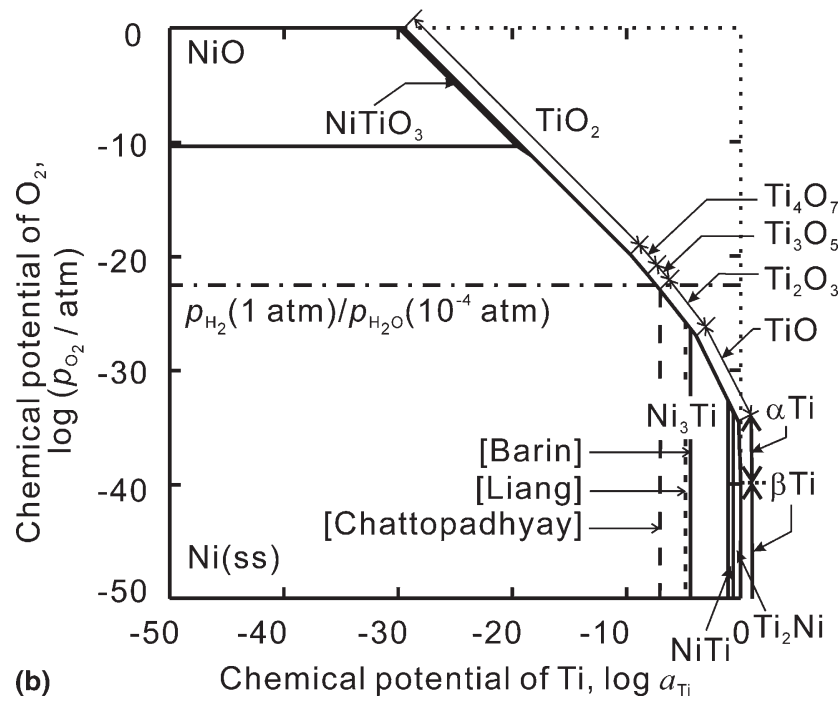

FIG. 3. (a) Chemical potential diagram of Ti-Ni-O system at $1000{ }^{\circ} \mathrm{C}$. (b) Projection view to $\log p_{\mathrm{O}_{2}}-\log a_{\mathrm{Ti}}$ plane. [Barin], [Liang], and [Chattopadhyay] correspond to the phase boundary line between $\mathrm{Ni}(\mathrm{ss})$ and $\mathrm{Ni}_{3} \mathrm{Ti}$ calculated with the reported Gibbs energies of formation of $\mathrm{Ni}_{3} \mathrm{Ti}$. Dashed line shows the chemical potential of oxygen in equilibrium with hydrogen gas of $1 \mathrm{~atm}$ and water of $10^{-4} \mathrm{~atm}$. 
and $\beta$-Ti are considered to be solutions of titanium and oxygen, and $\mathrm{Ni}(\mathrm{ss})$ is considered to be a solution of nickel and titanium. The thermodynamic information of such solid solution was taken from the literature. ${ }^{11-13}$ Three different Gibbs energies of formation are reported for $\mathrm{Ni}_{3} \mathrm{Ti}$. [Barin], [Liang], and [Chattopadhyay] correspond to the phase boundary line between $\mathrm{Ni}(\mathrm{ss})$ and $\mathrm{Ni}_{3} \mathrm{Ti}$ calculated with the thermodynamic data described by Barin, ${ }^{12}$ Liang et al., ${ }^{13}$ and Chattopadhyay et al., ${ }^{11}$ respectively. From this figure, if we can reduce the partial pressure of oxygen to allow the formation of $\mathrm{TiO}, \mathrm{Ni}_{3} \mathrm{Ti}$ can be obtained in the presence of nickel. The dependency of the chemical potential of oxygen on temperature is shown in Fig. 1(b). There is a possibility that $\mathrm{TiO}$ forms at $1000{ }^{\circ} \mathrm{C}$ as reported by Huet et al. ${ }^{10}$

\section{EXPERIMENTAL}

Rutile type $\mathrm{TiO}_{2}$ powder (99.9\%, Wako Pure Chemical Industries, Ltd., Osaka, Japan) and Ni powder (catalyst grade, Fukuda Metal Foil and Powder Co., Ltd., Kyoto, Japan) were mixed at an atomic ratio of 1 to 3 by ball milling for $8 \mathrm{~h}$. The mixed powder was then pressed at 412 or $103 \mathrm{MPa}$ into the pellet. Reduction experiments were carried out using these sample pellets. The size of the sample pellets and experimental conditions are listed in Table I. P1 was reduced at $800^{\circ} \mathrm{C}$ by low-temperature hydrogen plasma generated with radio frequency (rf) plasma equipment under the $p_{\mathrm{H}_{2}}$ of $10^{-3}$ atm for $6 \mathrm{~h}$. F1 and F2 are the reduction experiments by supercooled monatomic hydrogen. In this study, the hotwire method was adopted for making supercooled monatomic hydrogen. Figure 4 shows a schematic illustration of the experimental apparatus. Hydrogen gas was generated by electrolysis of deionized water and was purified with a permeating palladium membrane. Purified molecular hydrogen was then introduced from the top of the reactor, which was made from stainless steel, SUS316, and protected from thermal oxidation by argon gas. A part of the molecular hydrogen was dissociated to monatomic hydrogen by a tungsten filament heated at 2000 or $2500{ }^{\circ} \mathrm{C}$ by supplying direct current (dc) from the dc source. The filament temperature was measured by infrared radio thermometer. The sample temperature was maintained at $1000{ }^{\circ} \mathrm{C}$ in the stainless steel tube. Reduction experiments by atmospheric hydrogen gas, U1 and $\mathrm{U} 2$, were also carried out using the same apparatus. In these cases, the filament was not heated.

The product phase of each sample pellet after reduction was identified by a $2 \theta / \theta$ scan of x-ray diffraction (XRD; RINT2200, $\mathrm{Cu} \mathrm{K}$, Rigaku Corporation, Tokyo, Japan). The product phase in the surface layer was identified by grazing incidence XRD (GI-XRD; $\mathrm{Cu} \mathrm{K}{ }_{\alpha}$, X'Pert MRD, PANalytical, Almelo, The Netherlands), where the incident angle of $\mathrm{x}$-ray against the surface of

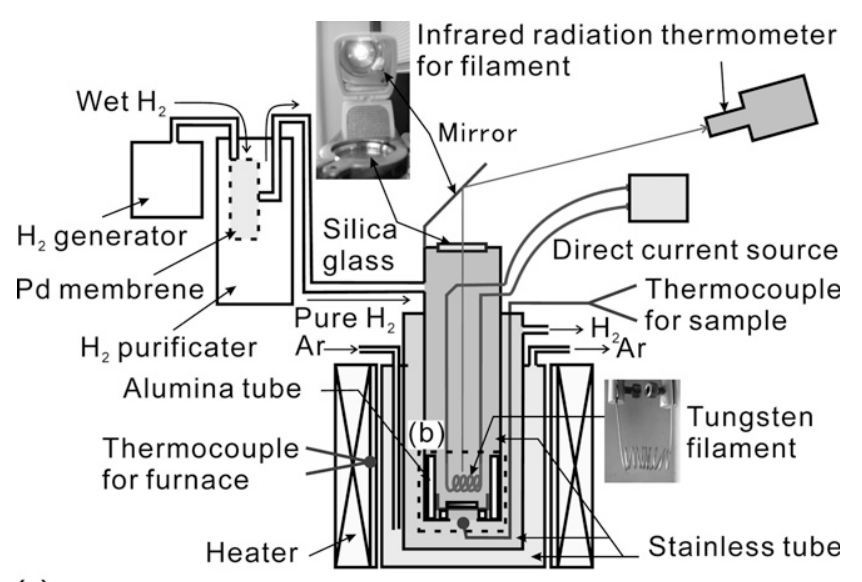

(a)

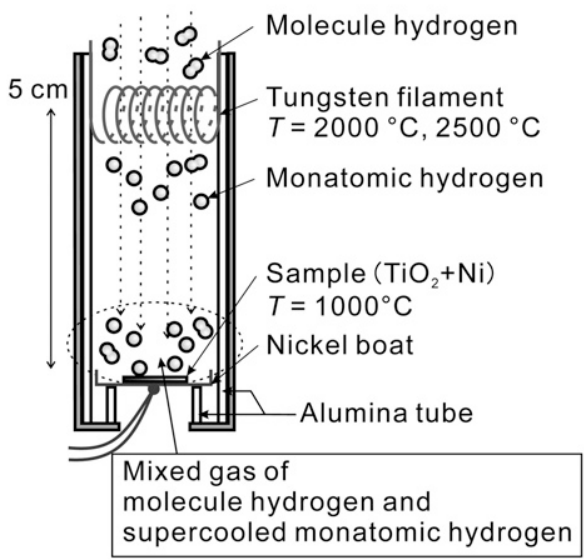

(b)

FIG. 4. (a) Schematic illustration of experimental apparatus for reduction by supercooled monatomic hydrogen and (b) close-up view around filament and sample shown by dashed line in (a).

TABLE I. Sample pellet prepared for the experiments and the reduction conditions.

\begin{tabular}{|c|c|c|c|c|c|c|c|c|}
\hline \multirow[b]{2}{*}{ Sample } & \multicolumn{3}{|c|}{ Sample pellet } & \multirow[b]{2}{*}{$\begin{array}{l}\text { Weight, } \\
w / \mathrm{mg}\end{array}$} & \multirow[b]{2}{*}{ Reductant } & \multicolumn{2}{|c|}{ Temperature, $T /{ }^{\circ} \mathrm{C}$} & \multirow[b]{2}{*}{$\begin{array}{c}\text { Treatment } \\
\text { time, } t / \mathrm{h}\end{array}$} \\
\hline & $\begin{array}{c}\text { Pressure, } \\
p / \mathrm{MPa}\end{array}$ & $\begin{array}{l}\text { Diameter, } \\
r / \mathrm{mm}\end{array}$ & $\begin{array}{c}\text { Thickness, } \\
d / \mathrm{mm}\end{array}$ & & & Sample & Filament & \\
\hline $\mathrm{P} 1$ & 103 & 11 & 2.4 & $8.4 \times 10^{2}$ & Low temperature hydrogen plasma & 800 & - & 6 \\
\hline $\mathrm{F} 1$ & 412 & 11 & 0.2 & $7.0 \times 10$ & Supercooled monatomic hydrogen & 1000 & 2000 & 12 \\
\hline $\mathrm{F} 2$ & 412 & 11 & 0.4 & $2.5 \times 10^{2}$ & Supercooled monatomic hydrogen & 1000 & 2500 & 12 \\
\hline U1 & 412 & 11 & 0.3 & $1.2 \times 10_{2}$ & Atmospheric hydrogen gas & 800 & - & 6 \\
\hline $\mathrm{U} 2$ & 412 & 11 & 0.2 & $7.1 \times 10$ & Atmospheric hydrogen gas & 1000 & - & 12 \\
\hline
\end{tabular}


the sample pellet was $1^{\circ}$. The composition in the nickel phase was measured by energy-dispersive $\mathrm{x}$-ray spectrometer (EDX; JED-2300, JEOL, Tokyo, Japan) with field-emission scanning electron microscopy (FE-SEM; JSM-6500F, JEOL).

\section{RESULTS}

\section{A. Reduction by low-temperature hydrogen plasma}

The photographs and SEM image of the sample pellet before and after the reduction experiment at $800{ }^{\circ} \mathrm{C}$ by low-temperature hydrogen plasma (P1) and atmospheric hydrogen gas (U1) are shown in Fig. 5. The color of the front surface of the sample pellet of P1 was changed from gray to black. This implies that a change occurred on the surface. Although it was observed that the grains slightly grew after the reduction from the SEM image, the grains were too small to measure the composition of each grain by EDX. The XRD profiles of the samples before and after the reduction experiment at $800{ }^{\circ} \mathrm{C}$ (U1, P1) were shown in Fig. 6. The peaks of $\mathrm{TiO}_{2}$ and $\mathrm{Ni}$ were observed in all diffraction profiles. However, although the color of the surface was changed in the sample of P1, there was no evidence for the reaction of the surface in the XRD profile. Consequently, the surface of the sample pellet P1 was analyzed by GI-XRD and the results are also shown in Fig. 6. The diffraction peaks of $\mathrm{Ti}_{2} \mathrm{O}_{3}$, which is lower titanium oxide, were observed in the diffraction profile. Thus, we confirmed that $\mathrm{Ti}_{2} \mathrm{O}_{3}$ formed on the surface of the sample after the reduction by low-temperature hydrogen plasma.

\section{B. Reduction by supercooled monatomic hydrogen}

Figure 7 shows the results of XRD analysis before and after the reduction experiments at $1000{ }^{\circ} \mathrm{C}$ by atmospheric hydrogen gas (U2) and supercooled monatomic hydrogen (F1 and F2). It was found by XRD that $\mathrm{Ti}_{3} \mathrm{O}_{5}$ formed after the reduction by supercooled monatomic hydrogen under a filament temperature of 2000 and $2500{ }^{\circ} \mathrm{C}$, whereas $\mathrm{Ti}_{4} \mathrm{O}_{7}$ formed after the reduction by atmospheric hydrogen gas. This indicates that supercooled monatomic hydrogen is a stronger reductant than atmospheric hydrogen gas. Figure 8 shows backscattered electron images of the samples after reduction. White and gray corresponded to nickel and titanium oxide,

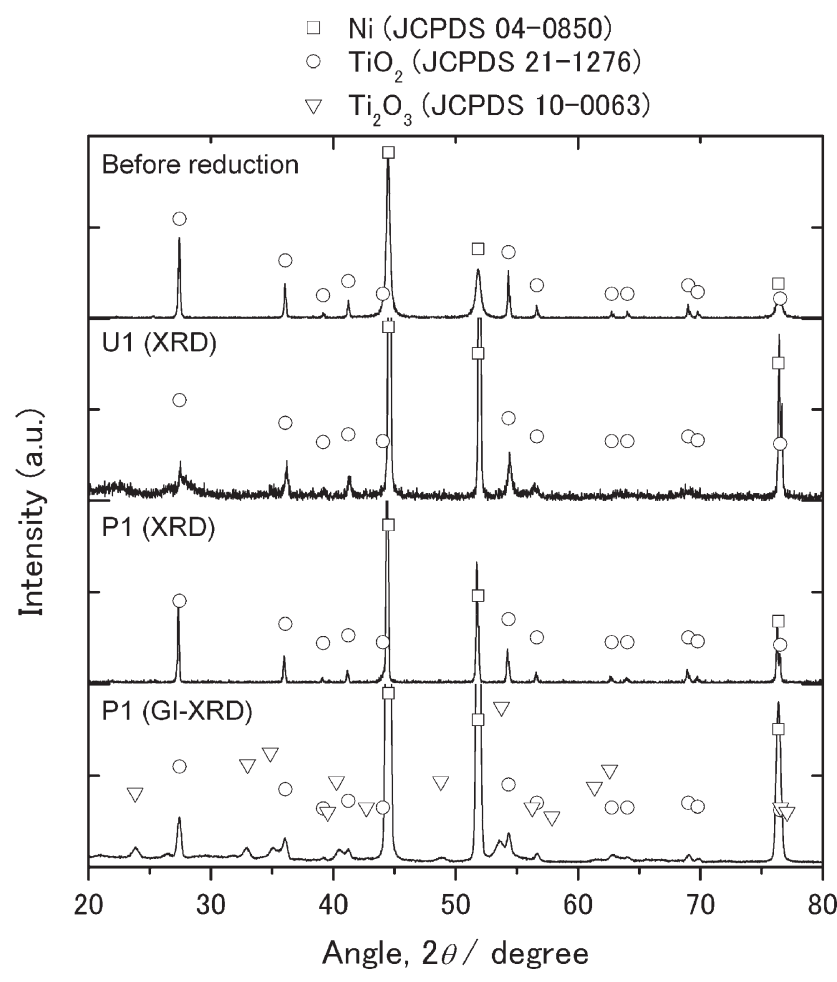

FIG. 6. XRD and GI-XRD profiles of the sample pellet before and after the reduction experiment by low-temperature hydrogen plasma. Symbols show peak positions referred from JCPDS card.

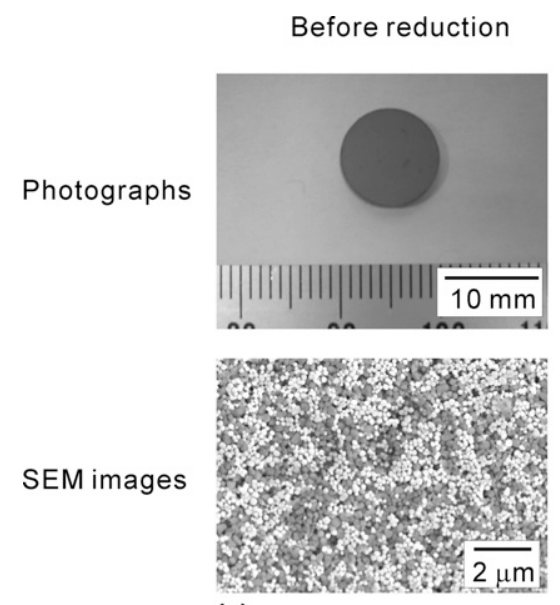

(a)

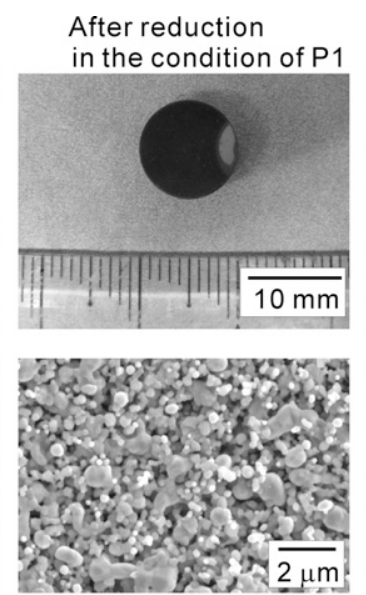

(b)

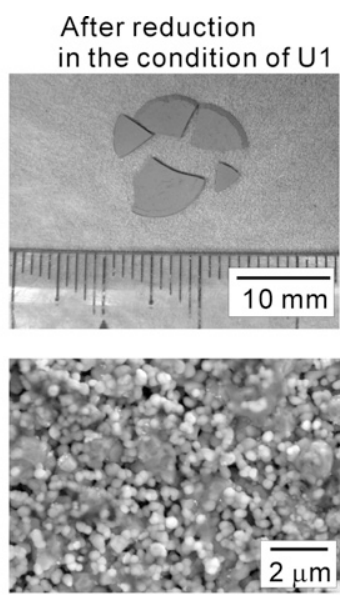

(c)

FIG. 5. Photographs and SEM images of the in the reduction experiment by hydrogen plasma. (a) Sample before reduction. (b) Sample reduced at $800{ }^{\circ} \mathrm{C}$ by low-temperature hydrogen plasma (P1). (c) Sample reduced at $800{ }^{\circ} \mathrm{C}$ by atmospheric hydrogen gas (U1). 
respectively, by EDX analysis. The grain size is almost identical in all samples, which implies that the temperature of the surface was maintained at around $1000^{\circ} \mathrm{C}$ in these three experiments. The concentration of titanium in $\mathrm{Ni}(\mathrm{ss})$ was measured by EDX and the results are listed in Table II. The concentration is almost the same in all samples, and independent of the filament temperature. Here, the solubility of titanium is different in $\mathrm{Ni}(\mathrm{ss})$ in equilibrium with $\mathrm{Ti}_{3} \mathrm{O}_{5}$ or $\mathrm{Ti}_{4} \mathrm{O}_{7}$, which are the product phases after the reduction by supercooled monatomic hydrogen or atmospheric hydrogen, respectively. Never-
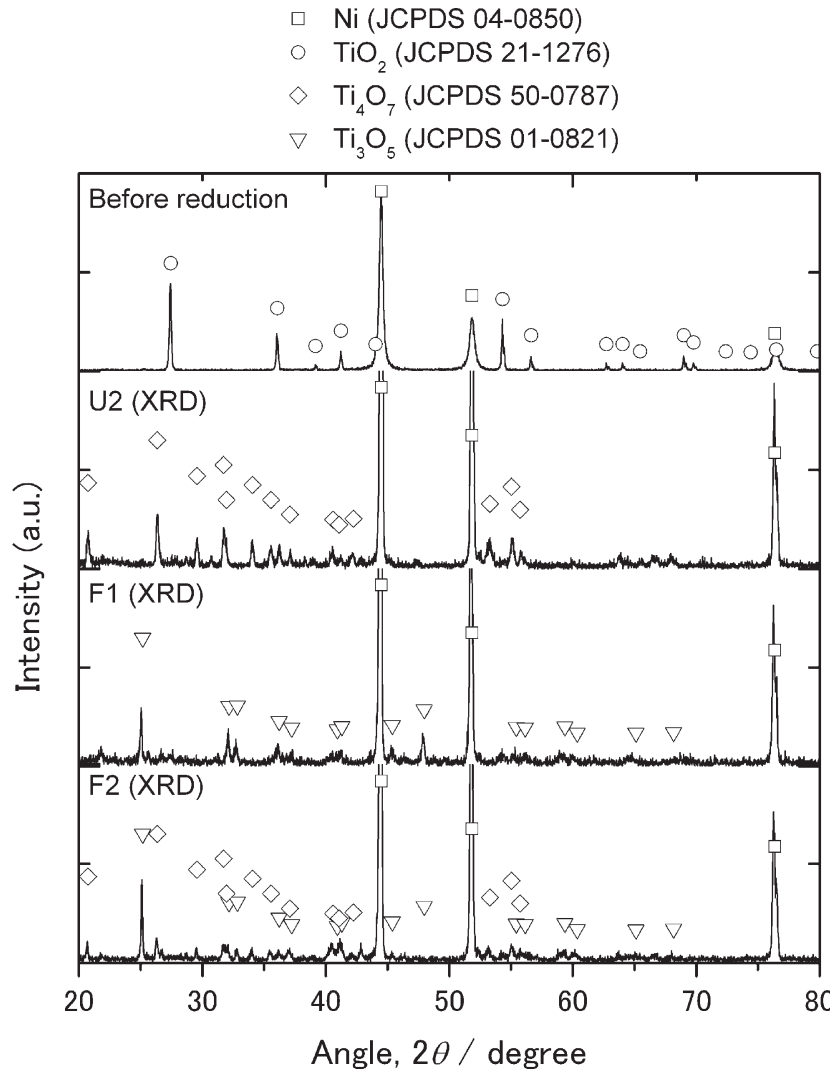

FIG. 7. XRD profiles of the reduction experiment with supercooled monatomic hydrogen. Symbols shows peak positions referred from JCPDS card. U2 is the sample reduced at $1000{ }^{\circ} \mathrm{C}$ by atmospheric hydrogen gas. F1 and F2 are the samples reduced at $1000{ }^{\circ} \mathrm{C}$ by supercooled monatomic hydrogen generated at 2000 and $2500{ }^{\circ} \mathrm{C}$, respectively. theless, such a difference of concentration of $\mathrm{Ti}$ in $\mathrm{Ni}(\mathrm{ss})$ was not observed in EDX analysis. This contradiction is discussed in the next section.

\section{DISCUSSION}

\section{A. Difference of surface and bulk in the reduction by supercooled monatomic hydrogen}

In the experiment of $\mathrm{U} 2$, although the concentration of Ti in Ni(ss) by EDX was 1.8 at.\%, the titanium oxide identified by XRD was $\mathrm{Ti}_{4} \mathrm{O}_{7}$, which must be in equilibrium with pure Ni, as shown in Fig. 2. This contradiction between the experimental results and the phase diagram of $\mathrm{Ti}-\mathrm{Ni}-\mathrm{O}$ system can be explained by the difference of detectable depth between EDX and XRD. The detectable depth of EDX depends on the penetration depth of electron beam, $L_{\mathrm{e}}$, which is represented as follows ${ }^{14}$ :

$$
L_{\mathrm{e}}=5.025 \times 10^{-12} \frac{A E_{0}^{5 / 3}}{\lambda_{\mathrm{s}} \rho Z^{8 / 9}} .
$$

Here, $A, E_{0}, \lambda_{\mathrm{s}}, \rho$, and $Z$ are atomic weight, incident energy of electron beam, density, empirical constant, and atomic number, respectively. Since we measured the concentration of $\mathrm{Ti}$ in $\mathrm{Ni}(\mathrm{ss})$, we considered the penetration of electron beam into $\mathrm{Ni}$. The $\lambda_{\mathrm{s}}$ is 0.182 when $E_{0}$ is $10 \mathrm{keV}$. Under this condition, $L_{\mathrm{e}}$ is calculated to be $0.44 \mu \mathrm{m}$.

On the other hand, the detectable depth of XRD is estimated from the effective penetration depth of $\mathrm{x}$-rays, $L_{\mathrm{X}}$, which can be calculated using the following equation, ${ }^{15}$

$$
L_{\mathrm{X}}=\frac{\sin \theta}{2 \mu} \ln \frac{1}{1-G}
$$

TABLE II. Product phases and concentration of titanium in Ni(ss) after the reduction experiments.

\begin{tabular}{lll}
\hline \hline \multicolumn{1}{c}{ Sample } & \multicolumn{1}{c}{ Product phase } & Concentration of Ti in N(ss) (at.\%) \\
\hline P1 (bulk) & $\mathrm{Ni}, \mathrm{TiO}_{2}$ & Not observed \\
P1 (surface) & $\mathrm{Ni}, \mathrm{Ti}_{2} \mathrm{O}_{3}, \mathrm{TiO}_{2}$ & Not observed \\
$\mathrm{F} 1$ (bulk) & $\mathrm{Ni}, \mathrm{Ti}_{3} \mathrm{O}_{5}$ & $1.6 \pm 0.4$ \\
$\mathrm{~F} 2$ (bulk) & $\mathrm{Ni}, \mathrm{Ti}_{3} \mathrm{O}_{5}, \mathrm{Ti}_{4} \mathrm{O}_{7}$ & $1.9 \pm 0.4$ \\
$\mathrm{U} 1$ (bulk) & $\mathrm{Ni}, \mathrm{TiO}_{2}$ & Not observed \\
$\mathrm{U} 2$ (bulk) & $\mathrm{Ni}, \mathrm{Ti}_{4} \mathrm{O}_{7}$ & $1.8 \pm 0.5$ \\
\hline \hline
\end{tabular}

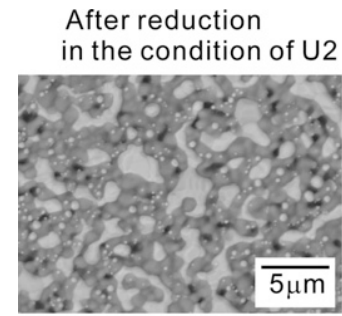

(a)

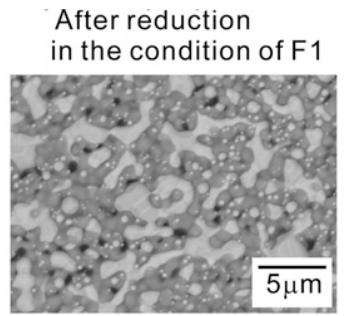

(b)

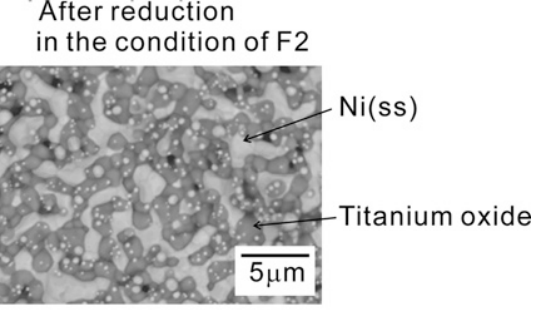

(c)

FIG. 8. Backscattered electron images after the reduction experiment by supercooled monatomic hydrogen. 
Here, $\theta$ and $\mu$ are the incident angle of $\mathrm{x}$-ray and the $\mathrm{x}$-ray linear absorption coefficient, respectively. $G$ is the ratio of the intensity of the diffracted x-ray from the surface region to the total intensity of the diffracted x-ray. Thus, $G$ shows the ratio of the information from the surface region of a depth to that from the whole sample in the XRD profile. We regarded the effective penetration depth as the depth giving $99 \%$ of the information in XRD profiles. When $G$ is 0.99 , the effective penetration depth of $\mathrm{x}$-rays is $7.4 \mu \mathrm{m}$, where $\theta$ is $10^{\circ}$. This is the case where the effective penetration depth is the shallowest. In fact, when $\theta$ is $40^{\circ}, L_{\mathrm{x}}$ is calculated to be $27 \mu \mathrm{m}$. Thus, we can obtain only the information of the thin layer on the surface region by EDX. Therefore, on the assumption that the titanium oxide formed after the reduction by atmospheric hydrogen gas at $1000{ }^{\circ} \mathrm{C}$ is the difference between surface and bulk, as shown in Fig. 9(a), we can then understand why the concentration of $\mathrm{Ti}$ in $\mathrm{Ni}$ (ss) measured by EDX implies the existence of $\mathrm{Ti}_{3} \mathrm{O}_{5}$, and $\mathrm{Ti}_{4} \mathrm{O}_{7}$ was observed by XRD in the sample of

\section{After reduction} in the condition of $U 2$

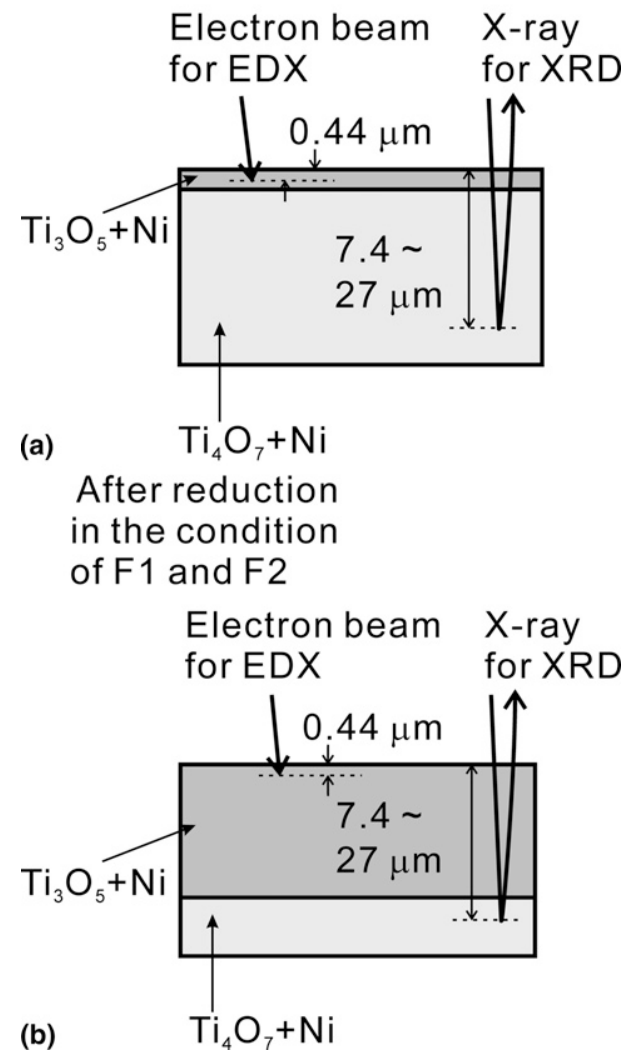

FIG. 9. Schematic illustration of the penetration of electron beam and x-ray. Gray layer consists of $\mathrm{Ti}_{3} \mathrm{O}_{5}$ and Ni. White layer consists of $\mathrm{Ti}_{4} \mathrm{O}_{7}$ and Ni. (a) Sample reduced at $1000^{\circ} \mathrm{C}$ by atmospheric hydrogen gas (U2). (b) Sample reduced at $1000{ }^{\circ} \mathrm{C}$ by supercooled monatomic hydrogen $(\mathrm{F} 1, \mathrm{~F} 2)$.
U2. Furthermore, we can reasonably assume that the thickness of the $\mathrm{Ti}_{3} \mathrm{O}_{5}$ layer of the samples after the reduction by supercooled monatomic hydrogen is wider than that by atmospheric hydrogen gas, as shown in Fig. 9. These assumptions might be true because the equilibrium phase with $\mathrm{H}_{2}$ and $\mathrm{H}_{2} \mathrm{O}\left(p_{\mathrm{H}_{2}}=1 \mathrm{~atm}, p_{\mathrm{H}_{2} \mathrm{O}}=\right.$ $\left.10^{-4} \mathrm{~atm}\right)$ is $\mathrm{Ti}_{3} \mathrm{O}_{5}$ in the chemical potential diagram of Fig. 3(b).

\section{B. Reduction mechanism of the reduction by nonequilibrium hydrogen gas}

Nonequilibrium hydrogen gas, including low-temperature hydrogen plasma and supercooled monatomic hydrogen, is sometimes treated as having a thermodynamically high reduction potential on the assumption of the local equilibrium between water and excited hydrogen. ${ }^{16}$ We think that the dominant exited hydrogen species is monatomic hydrogen. In Fig. 1(b), we plotted a line for $\mathrm{R} T \ln p_{\mathrm{O}_{2}}$ in equilibrium with $\mathrm{H}$ (monatomic hydrogen) of $1 \mathrm{~atm}$ and $\mathrm{H}_{2} \mathrm{O}$ of $1 \mathrm{~atm}$. But, considering the results of this study, we have to consider that kinetical reasons lead to such hypothetical high reduction ability in a low-temperature plasma process. The low-temperature hydrogen plasma we used is excited by an electromagnetic field, and supercooled monatomic hydrogen is excited by high temperature. As a result, nonequilibrium hydrogen gas is in a high energy state. This high energy state is recovered by reaction with the surface of the condensed substrate as a release of heat. Since the energy of nonequilibrium hydrogen gas is too high, multiple states of product surface can be allowed. The reacted surface should then move to equilibrium. We call these processes (1) the releasing energy process and (2) the relaxing process. These processes can be illustrated as in Fig. 10. In the releasing energy process, nonequilibrium hydrogen gas reacted with $\mathrm{TiO}_{2}$ accompanied with the removal of oxygen on the surface as water. This reaction leads to a lack of oxygen and formation of $\mathrm{Ti}_{2} \mathrm{O}_{3}$ or $\mathrm{Ti}_{3} \mathrm{O}_{5}$ or even more reduced titanium oxides, which are not in equilibrium. In the relaxing process, $\mathrm{Ti}_{2} \mathrm{O}_{3}$ or $\mathrm{Ti}_{3} \mathrm{O}_{5}$ then reacts with the water vapor in the gas boundary layer near the sample or with titanium oxide located deeper in the sample. As a result, the equilibrium products $\mathrm{TiO}_{2}$ or $\mathrm{Ti}_{4} \mathrm{O}_{7}$ are formed. When the relaxing process is slow, the nonequilibrium product remains and it has a higher energy than that in equilibrium. We think this is the case for the reduction by low-temperature plasma at $800{ }^{\circ} \mathrm{C}$. In the case of reduction at $1000{ }^{\circ} \mathrm{C}$, the relaxing process is fast. We see that the product is the same with atmospheric hydrogen gas, although the thickness is different. Actually, we can also see a similar process in plasma nitridation. The reaction rate of plasma nitridation is, as is well known, faster than that of normal nitridation using $\mathrm{NH}_{3} \cdot{ }^{17}$ Using plasma nitridation, many metastable 


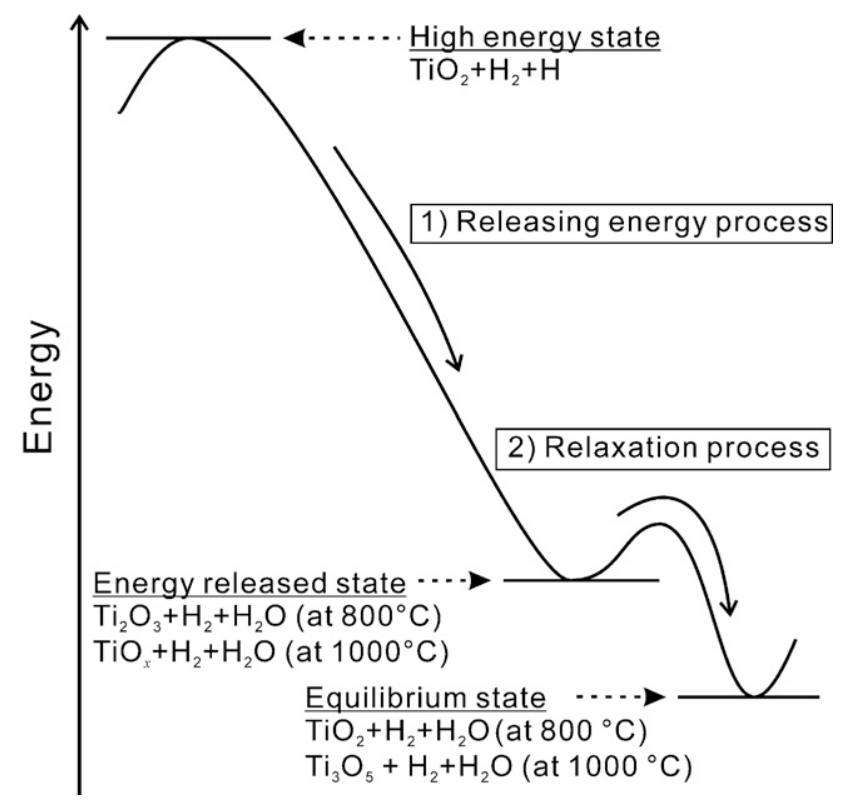

FIG. 10. Reaction processes of the reduction by nonequilibrium hydrogen gas. (1) shows the releasing process and (2) shows the relaxation process.

phases can be obtained on the surface of the substrate at low temperature. This metastable phase decreases through the diffusion of nitrogen. ${ }^{18}$ The former and latter reactions correspond to the releasing and relaxation processes, respectively.

For the difference of thickness of $\mathrm{Ti}_{3} \mathrm{O}_{5}$ at reduction experiments of $1000{ }^{\circ} \mathrm{C}$, we think that the supercooled monatomic hydrogen enhanced the reduction rate on the surface. Kondo et al. ${ }^{19}$ reported on the reduction of $\mathrm{CuO}$ to metallic $\mathrm{Cu}$ by molecular hydrogen at $450{ }^{\circ} \mathrm{C}$ and by supercooled monatomic hydrogen under $150{ }^{\circ} \mathrm{C} .{ }^{18}$ The value of the standard Gibbs energy change of the reaction expressed below is $-97.8 \mathrm{~kJ} \mathrm{~mol}^{-1}$ at $450{ }^{\circ} \mathrm{C}$ :

$$
\mathrm{CuO}+\mathrm{H}_{2} \rightarrow \mathrm{Cu}+\mathrm{H}_{2} \mathrm{O} .
$$

Even at $150{ }^{\circ} \mathrm{C}$, it is negative and $-87.7 \mathrm{~kJ} \mathrm{~mol}^{-1}$. In the case of the reduction by molecular hydrogen, this reaction did not proceed despite the negative Gibbs energy. However, metallic $\mathrm{Cu}$ was obtained by the reduction using supercooled monatomic hydrogen at around $150{ }^{\circ} \mathrm{C}$. These results also indicate that the reduction rate on the surface is kinetically enhanced by supercooled monatomic hydrogen.

\section{CONCLUSIONS}

In this study, we investigated the reduction behaviors of $\mathrm{TiO}_{2}$ in the presence of $\mathrm{Ni}$ by atmospheric hydrogen gas and nonequilibrium hydrogen gas, including lowtemperature hydrogen plasma and supercooled monatomic hydrogen. Although we could not obtain $\mathrm{Ni}_{3} \mathrm{Ti}$ as originally planned, we obtained some fundamental knowledge about the reaction mechanism of the reduction by nonequilibrium hydrogen gas. It was clear that nonequilibrium hydrogen gas has a higher reduction potential than atmospheric hydrogen gas. The reduction ability of low-temperature hydrogen plasma, by which $\mathrm{TiO}_{2}$ was reduced to $\mathrm{Ti}_{2} \mathrm{O}_{3}$, and that of supercooled monatomic hydrogen, by which $\mathrm{TiO}_{2}$ was reduced to $\mathrm{Ti}_{3} \mathrm{O}_{5}$, is only kinetically higher.

\section{ACKNOWLEDGMENTS}

This work was partly supported by the JFE 21 st Century Foundation. Enlightening discussion on supercooled monatomic hydrogen and hot-wire method with Prof. Kiyoshi Yasutake and Prof. Hiromasa Ohmi (Osaka University, Japan) is gratefully acknowledged. The authors also expressed their deep acknowledgment to Prof. Mitsuda at the University of Tokyo.

\section{REFERENCES}

1. M. Hagiwara: New evolution in titanium research and development in Japan. J. Jpn. Inst. Light Metals 55, 532 (2005).

2. R.M. Scanlan, A.P. Malozemoff, and D.C. Larbalestier: Superconducting materials for large scale applications. Proc. IEEE 92, 1939 (2004).

3. G-Z. Chen, D.J. Fray, and T.W. Farthing: Direct electrochemical reduction of titanium dioxide to titanium in molten calcium chloride. Nature 401, 361 (2000).

4. T. Uda and T.H. Okabe: Reduction process of titanium oxide using molten salt. Titanium Jpn. 50, 325 (2002).

5. T.H. Okabe, R.O. Suzuki, T. Oishi, and K. Ono: Thermodynamic properties of dilute titanium-oxygen solid solution in beta phase. Mater. Trans., JIM 32, 485 (1991).

6. P.J. Meschter and W.L. Worrel: An investigation of high-temperature thermodynamic properties in the $\mathrm{Pt}-\mathrm{Ti}$ system. Metall. Trans. A 7, 299 (1976).

7. R. Shioi, S. Imashuku, T. Uda, and Y. Awakura: Reduction of titanium oxide in the presence of platinum and consideration of a new smelting method for titanium, in Abstracts of the Spring Meeting for the Mining and Material Processing Institute of Japan, Vol. 89 (2006).

8. D.E. Bullard and D.C. Lynch: Reduction of titanium dioxide in a nonequilibrium hydrogen plasma. Metall. Mater. Trans. B 28. 1069 (1997).

9. Y-W. Zhang, W-Z. Ding, X-G. Lu, S-Q. Guo, and K-D. Xu: Reduction of $\mathrm{TiO}_{2}$ with hydrogen cold plasma in DC pulsed glow discharge. Trans. Nonferrous Met. Soc. China 15, 594 (2005).

10. S. Huet, T. Belmonte, J.M. Thiébaut, S. Bockel, and H. Michel: Reduction of $\mathrm{TiO}_{2}$ assisted by a microwave plasma at atmospheric pressure. Thin Solid Films 475, 63 (2005).

11. G. Chattopadhyay and H. Kleykamp: Phase equilibria and thermodynamic studies in the titanium-nickel and titanium-nickeloxygen systems. Z. Metallkd. 74, 182 (1983).

12. I. Barin: Thermodynamic Data of Pure Substances, 3rd ed. (Wiley-VCH, Weinheim, Germany, 1995).

13. H-Y. Liang and Z-P. Jin: A reassessment of the Ti-Ni system. Calphad 17, 415 (1993).

14. K. Kaneya and S. Okayama: Penetration and energy-loss theory of electrons in solid targets. J.Phvs. D: Appl. Phvs. 5, 43 (1982). 
15. B.D. Cullity: Elements of X-ray Diffraction, 2nd ed. (AddisonWesley Publishing Co., Reading, MA, 1978).

16. K.T. Jacob, A. Chandra, and R.M. Mallya: An assessment of the reduction potential of hydrogen plasma. Z. Metallkd. 91, 401 (2005).

17. K. Ichii, A. Nishimoto, K. Nakao, and K. Akamatsu: Low temperature nitriding of austenitic stainless steel. J. Surf. Fin. Soc. Japan 54, 200 (2003).
18. A. Straboni, L. Pichon, and T. Girardeau: Production of stable and metastable phases of zirconium nitrides by $\mathrm{NH}_{3}$ plasma nitridation and by double ion beam sputtering of zirconium films. Surf. Coat. Technol. 125, 100 (2005).

19. E. Kondoh, M. Fukasawa, and T. Ojimi: Reduction of thin oxidized copper films using a hot-filament hydrogen radical source. J. Vac. Sci. Technol., A 25, 415 (2007). 